\title{
Visualizing movement in the tumor microenvironment landscape
}

\author{
Veronica Calvo and Paraic A Kenny*
}

\begin{abstract}
The local microenvironment influences tumor progression in several important ways. A recent study by Nguyen-Ngoc and colleagues used explants of primary human and mouse mammary tumors to examine how the composition of the extracellular matrix modulates tumor cell invasion. Culture in the presence of a three-dimensional laminin-rich basement membrane, similar to the mammary basement membrane in vivo, resulted in minimal invasion. In contrast, identical tumor fragments in a collagen I matrix which resembles the interstitial breast stroma exhibited a pronounced protrusive migration and local dissemination. These data emphasize the importance of the laminin-rich basement membrane in constraining tumor cell invasion.
\end{abstract}

The transition from carcinoma in situ to invasive disease is a pivotal event in the natural history of breast cancer and has a pronounced effect on the prognosis of the patient with cancer. Of particular significance is the efficiency with which the invasive tumor cells disseminate locally and to distant sites. It has become clear that, though indisputably important, the accumulation of mutations in key genes is necessary but not sufficient for full expression of the malignant phenotype. The influence of collaborating microenvironmental factors on this transition is an area of intense investigation. A considerable body of work has illuminated the role of the tumorassociated macrophage in eliciting invasive behavior [1]. Less studied has been the impact of the precise composition of the local tissue extracellular matrix on modulating breast cancer cell motility and dissemination. The basement membrane of non-malignant mammary epithelium is a thin layer of extracellular matrix

*Correspondence: paraic.kenny@einstein.yu.edu

Department of Developmental \& Molecular Biology, Albert Einstein College of Medicine, 1300 Morris Park Avenue, Bronx, NY 10461, USA composed primarily of laminins, collagen IV, nidogen, entactin, and proteoglycans [2], whereas the interstitial matrix beyond the mammary ducts is composed of collagen I. The basement membrane acts as a barrier to local tissue invasion but, once this barrier is breached, does the surrounding microenvironment simply provide a passive landscape through which the neoplastic cells can rampage, or do factors intrinsic to this microenvironment actively promote the invasive migratory behavior of these cells?

Using time-lapse microscopy, Nguyen-Ngoc, Ewald, and colleagues [3] recently examined the impact of local extracellular matrix composition on regulating protrusive migration and cell dissemination. Epithelial fragments from either primary human or mouse mammary carcinomas cultured in a reconstituted basement membrane matrix (Matrigel) either were indolent or only occasionally exhibited a multilayered collective migration. In contrast, fragments explanted into collagen I gels showed extensive protrusive migration and cell dissemination. Dissemination was primarily mesenchymal, although amoeboid migration was also frequently observed. Collective dissemination of groups of cells was seen in a minority of cases. Isolation of organoids from one substrate and regrowth on the reciprocal matrix indicated that these changes in invasion mode are reversible. The inability of the malignant cells in collagen gels to deposit a laminin basement membrane, reminiscent of a much earlier study [4], was a major distinguishing feature between malignant and normal cells in this assay. Surprisingly, attempts to define matrix-dependent gene expression profiles by microarray analysis revealed minimal differences between Matrigel- and collagen Icultured tumor fragments, suggesting that key mRNA differences may be restricted to a minority of cells or that the important alterations are post-translational.

Several questions arise from this interesting study. Although a small number of primary human tumor specimens were used, the bulk of the analysis was performed with tumor explants from MMTV-PyMT mice, and MMTV-PyMT is an oncogene that does not have a straightforward parallel in human breast cancer. It would be interesting to expand this approach to 
additional models to determine the extent to which these findings can be generalized. Previous work from the Pearson laboratory [5] has indicated that fibroblastdependent remodeling of collagen is key to inducing invasion in mixed cultures of Matrigel/collagen I and that basal, but not luminal, breast cancer cell lines preferentially invade under those conditions. In the clinic, cases of invasive breast cancer are frequently observed without lymph node metastasis. Almost all of the human tumors tested in this study were from node-positive patients and thus had already demonstrated a strong capacity for invasive migration in the original patient. Performing similar experiments with tumor explants from nodenegative patients would help determine whether the migratory activity of both node-negative and -positive tumors is similarly influenced by the local extracellular matrix environment.

A significant strength of this study is the extensive body of time-lapse imaging following the behavior of the explants for several days, and this allowed the quantification of disseminating cells and the analysis of their modes of invasion. It is striking how rare the disseminating cells are (only 612 disseminating cells from PyMT tumor fragments were observed in a total of 48 multi-day movies). This is consistent with an intravital imaging study of MMTV-PyMT tumors, which showed that motile cells comprise a very small proportion of the tumor bulk [6]. Given the relative rarity of migratory cells, it is perhaps not surprising that gene expression profiling of the bulk tumor fragments did not reveal a substantial number of differentially expressed genes between the two matrix environments. This issue highlights the value of selectively isolating and profiling the invasive tumor cells, an approach recently reported in vivo by Patsialou and colleagues [7]. The nature of the mechanisms conferring a highly motile phenotype on some tumor cells while their neighbors remain indolent is another important question that remains to be resolved.

Finally, the use of three-dimensional extracellular matrix culture models - usually laminin-rich matrices like Matrigel - for mammary gland biology and breast cancer research has undergone a rapid expansion over the past decade [8-10]. A provocative question raised by this study is whether our experimental approaches to study invasive breast cancer cells might more appropriately be directed toward three-dimensional collagen I cultures.

\section{Competing interests}

The authors declare that they have no competing interests.

\section{Acknowledgments}

The work in the authors' laboratory is supported by grants from Susan G. Komen for the Cure (KG100888) and the American Cancer Society (RSG-12267-01-TBE) to PK and by a postdoctoral fellowship from Fundacion Alfonso Martin Escudero to VC.

Published: 15 January 2013

\section{References}

1. Qian BZ, Pollard JW: Macrophage diversity enhances tumor progression and metastasis. Cell 2010, 141:39-51.

2. Erickson AC, Couchman JR: Still more complexity in mammalian basement membranes. J Histochem Cytochem 2000, 48:1291-1306.

3. Nguyen-Ngoc KV, Cheung KJ, Brenot A, Shamir ER, Gray RS, Hines WC, Yaswen $P$, Werb Z, Ewald AJ: ECM microenvironment regulates collective migration and local dissemination in normal and malignant mammary epithelium. Proc Natl Acad Sci U S A 2012, 109:E2595-2604.

4. David G, Van der Schueren B, Bernfield M: Basal lamina formation by normal and transformed mouse mammary epithelial cells duplicated in vitro. J Nat/ Cancer Inst 1981, 67:719-728.

5. Dang TT, Prechtl AM, Pearson GW: Breast cancer subtype-specific interactions with the microenvironment dictate mechanisms of invasion. Cancer Res 2011, 71:6857-6866.

6. Wang W, Wyckoff JB, Goswami S, Wang Y, Sidani M, Segall JE, Condeelis JS: Coordinated regulation of pathways for enhanced cell motility and chemotaxis is conserved in rat and mouse mammary tumors. Cancer Res 2007, 67:3505-3511.

7. Patsialou A, Wang Y, Lin J, Whitney K, Goswami S, Kenny PA, Condeelis JS: Selective gene expression profiling of migratory tumor cells in vivo predicts clinical outcome in breast cancer patients. Breast Cancer Res 2012, 14:R139.

8. Debnath J, Brugge JS: Modelling glandular epithelial cancers in threedimensional cultures. Nat Rev Cancer 2005, 5:675-688.

9. Kenny PA, Lee GY, Myers CA, Neve RM, Semeiks JR, Spellman PT, Lorenz K, Lee EH, Barcellos-Hoff MH, Petersen OW, Gray JW, Bissell MJ: The morphologies of breast cancer cell lines in three-dimensional assays correlate with their profiles of gene expression. Mol Oncol 2007, 1:84-96.

10. Mroue R, Bissell MJ: Three-dimensional cultures of mouse mammary epithelial cells. Methods Mol Biol 2013, 945:221-250.

doi:10.1186/bcr3359

Cite this article as: Calvo V, Kenny PA: Visualizing movement in the tumor microenvironment landscape. Breast Cancer Research 2013, 15:301. 